\title{
Genome-wide analysis of DNA methylation identifies S100A13 as an epigenetic biomarker in individuals with chronic $(\geq 30$ years) type 2 diabetes without diabetic retinopathy
}

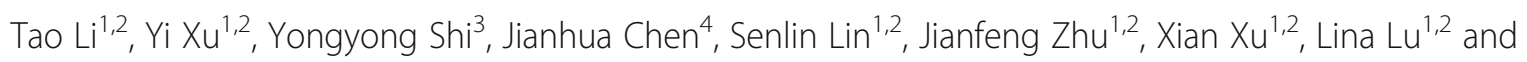
Haidong Zou ${ }^{1,2^{*}}$ (D)

\begin{abstract}
Background: This study aimed to determine the epigenetic biomarkers of diabetic retinopathy (DR) in subjects with type 2 diabetes mellitus (T2DM). This retrospective study is based on the Shanghai Xinjing community prevention and treatment administrative system of chronic diseases. The subjects enrolled herein were T2DM patients who had undergone long-term follow-up evaluation in the system. Two consecutive studies were conducted. In the discovery cohort, among 19 subjects who had developed DR with a DM duration < 3 years and 21 subjects without DR > 30 years after being diagnosed with DM, an Infinium Human Methylation 850 Beadchip was used to identify differential methylation regions (DMRs) and differential methylation sites (DMSs). The function of the genes was assessed through KEGG enrichment analysis, Gene Ontology (GO) analysis, and pathway network analysis. In the replication cohort, 87 DR patients with a short DM duration and 89 patients without DR over a DM duration > 20 years were compared to assess the association between DMSs and DR upon pyrosequencing.

Results: A total of 34 DMRs were identified. Genes containing DMSs with the top 5 highest beta value differences between DR and non-DR participants were located on chromosome 1 and were present in the S100A13 gene, which was associated with 71 GO terms. Two S100A13 gene sites, i.e., cg02873163 and cg11343894, displayed a good correlation with DR on pyrosequencing.
\end{abstract}

Conclusions: DMSs in the S100A13 gene may be potential biomarkers of DR.

Keywords: Diabetic retinopathy, T2DM, Biomarkers, DNA methylation, S100A13

\section{Background}

Epigenetic determinants reportedly contribute to the etiology of numerous systemic disorders such as diabetes

\footnotetext{
* Correspondence: zouhaidong@sjtu.edu.cn

${ }^{1}$ Shanghai Eye Diseases Prevention \& Treatment Center/Shanghai Eye Hospital, No. 380, Kangding Road, Shanghai 200040, China

${ }^{2}$ Department of Ophthalmology, Shanghai General Hospital, Shanghai Jiaotong University School of Medicine, Shanghai, China

Full list of author information is available at the end of the article
}

mellitus (DM) [1, 2]. Furthermore, hyperglycemia can affect DNA methylase activity or the methylation level of specific genes, which is the primary epigenetic modification [3-7]. Diabetic retinopathy (DR) is a common complication of DM and a leading cause of blindness among individuals aged 20-62 years in developed countries [8]. However, the exact etiology of DR remains unclear. Chen et al. reported that approximately 300 differential

C C The Author(s). 2020 Open Access This article is licensed under a Creative Commons Attribution 4.0 International License, which permits use, sharing, adaptation, distribution and reproduction in any medium or format, as long as you give appropriate credit to the original author(s) and the source, provide a link to the Creative Commons licence, and indicate if changes were made. The images or other third party material in this article are included in the article's Creative Commons licence, unless indicated otherwise in a credit line to the material. If material is not included in the article's Creative Commons licence and your intended use is not permitted by statutory regulation or exceeds the permitted use, you will need to obtain permission directly from the copyright holder. To view a copy of this licence, visit http://creativecommons.org/licenses/by/4.0/ The Creative Commons Public Domain Dedication waiver (http://creativecommons.org/publicdomain/zero/1.0/) applies to the data made available in this article, unless otherwise stated in a credit line to the data. 
methylation sites (DMSs) in DNA were associated with DR onset in type 1 DM (T1DM) patients [9]. Agardh et al. reported an association between DNA methylation and proliferative DR among individuals with T1DM [5]. However, among individuals with T2DM, no specific gene methylation levels have been reported to be associated with DR, even in PubMed.

In the present study, we assessed the epigenetic biomarkers of DR in T2DM patients. In the discovery cohort, among 19 subjects who developed DR with a DM duration of < 3 years and 21 subjects without DR > 30 years after being diagnosed with $\mathrm{DM},>850,000$ gene sites were assessed with a methylation chip. The functions of genes harboring DMSs with different degrees of methylation between the DR and non-DR participants were assessed through KEGG enrichment analysis, Gene Ontology (GO) analysis, and pathway network analysis and assessed for their association with DR. In the replication cohort, 87 DR patients with a short DM duration and 89 patients without DR over a DM duration > 20 years were compared to validate the association between the DMSs identified in the discovery cohort and DR through pyrosequencing.

\section{Results}

\section{Discovery study}

The basic and clinical characteristics of the $19 \mathrm{DR}$ and 21 non-DR subjects are summarized in Table 1. Except

Table 1 The basic and clinical characteristics of the 19 diabetic retinopathy patients (type 2 diabetes mellitus duration of $<3$ years) and 21 patients with no sign of diabetic retinopathy with a type 2 diabetes mellitus duration of $>30$ years enrolled in the discovery study

\begin{tabular}{llll}
\hline Characteristics* $^{*}$ & DR group & Non-DR group & $P$ value $^{\#}$ \\
\hline Number & 19 & 21 & $/$ \\
Sex (male) & 10 & 10 & 1 \\
Age (year) & $68.74 \pm 8.69$ & $74.19 \pm 8.59$ & 0.05 \\
Age of DM onset (year) & $67.26 \pm 8.59$ & $40.10 \pm 9.13$ & 0.00 \\
DM duration (year) & $1.47 \pm 1.07$ & $34.09 \pm 5.74$ & $/$ \\
Blood glucose (mmol/L) & $6.95 \pm 1.94$ & $6.99 \pm 2.22$ & 0.93 \\
HbA1c (\%) & $6.79 \pm 1.26$ & $7.53 \pm 1.44$ & 0.04 \\
BMI (kg/m $\left.{ }^{2}\right)$ & $25.30 \pm 4.55$ & $24.94 \pm 3.33$ & 0.94 \\
MAP (mmHg) & $102.98 \pm 12.53$ & $103.37 \pm 13.45$ & 0.93 \\
SER (D) & $-0.53 \pm 2.06$ & $0.17 \pm 2.01$ & 0.24 \\
IOP (mmHg) & $13.80 \pm 4.24$ & $15.02 \pm 2.62$ & 0.28 \\
AL (mm) & $23.23 \pm 1.13$ & $23.26 \pm 0.86$ & 0.93 \\
\hline
\end{tabular}

$D R$ diabetic retinopathy, T2DM type 2 diabetes mellitus

* $B M I$ body mass index; MAP $(\mathrm{mmHg})$ mean arterial pressure, equal to (systolic pressure $+2 *$ diastolic pressure)/3; SER (D) spherical equivalent rate, equal to spherical power $+1 / 2$ cylindrical power; IOP: intraocular pressure; AL: axial length.

${ }^{\#} X^{2}$ Chi-square test, $t$ Student's $t$ test, $Z$ Mann-Whitney test for differences in the duration and hemoglobin A1C (HbA1c) levels, the two groups did not significantly differ in age, sex, glucose levels, body mass index (BMI), spherical equivalent rate, intraocular pressure, or axial length. In the DR group, one had proliferative DR, 5 patients had severe nonproliferative DR, and 13 patients had moderate nonproliferative DR. Diabetic macular edema was detected in 3 patients. The 21 non-DR subjects did not present signs of DR on annual eye examination after they were diagnosed with T2DM. The duration of DM among these subjects was $>30$ years.

We identified thirty-four differential methylation regions (DMRs) when comparing the DR group and nonDR group. The DMRs comprised 11 hypermethylated regions and 23 hypomethylated regions. Of the 12 generelated sites, two DMSs were located at the transcriptional start site (TSS) 1500, one at TSS 200, one in the $5^{\prime}$-untranslated region UTR-5, three in EXON1, two in the gene body, and three in the 3 '-UTR (Table 2).

Global DNA methylation levels did not significantly differ between the DR group and the non-DR group $(P>$ 0.05). Furthermore, among 290 DMSs, methylation levels were decreased at 188 sites and increased at the other 102 sites in the DR group (Supplement 1). These DMSs were evenly distributed on all chromosomes (Fig. 1 ). The genes containing DMSs with the top 5 differences in beta values between DR and non-DR participants are listed in Table 3; these DMSs were detected in the S100A13 gene.

On GO enrichment analysis and KEGG pathway analysis (Supplement 2 and 3, respectively), the top 30 items are summarized in Fig. 2 a and b, respectively. DR-associated pathways included the Wnt signaling pathway, ubiquitin-mediated proteolysis, Tolllike receptor signaling pathway, tight junctions, the transforming growth factor-beta signaling pathway, the phosphatidylinositol signaling pathway, natural killer cell-mediated cytotoxicity, inositol phosphorylation metabolism, the hedgehog signaling pathway, aminoglycan degradation, Fcy receptor-mediated autophagy, cell adhesion factor signaling, the calcium signaling pathway, regulation of muscle contraction, neural tube formation, neural cell development, iron ion balance, fibroblast proliferation, phagocytosis associated with $\mathrm{Fc} \gamma$ receptor pathogenesis, glucosestimulated cellular responses, and developmental processes. Differential methylation in apoptotic responses and genes involved in AMPK activity are indicated in Fig. 2 a, b, and c. S100A13 was associated with $71 \mathrm{GO}$ terms, including signal transduction and calcium ion binding, which are reportedly associated with DR pathogenesis $[10,11]$. Therefore, the DMSs of the S100A13 gene were considered candidate DMSs. 
Table 2 Differential methylation regions corresponding genes and $\beta$ values of diabetic retinopathy and nondiabetic retinopathy subjects in the discovery study

\begin{tabular}{|c|c|c|c|c|c|}
\hline \multirow[t]{2}{*}{ DMR } & \multirow[t]{2}{*}{ Gene } & \multicolumn{3}{|l|}{$\boldsymbol{\beta}$ value } & \multirow[t]{2}{*}{$P$ value } \\
\hline & & DR group & Non-DR group & Difference & \\
\hline \multirow[t]{2}{*}{ TSS1500 } & MDFI & 0.414024 & 0.554308 & -0.140284 & 0.000962 \\
\hline & PGBD5 & 0.538043 & 0.365315 & 0.17273 & 0.000358 \\
\hline TSS200 & ADRA2C & 0.042839 & 0.27238 & -0.229541 & 0.002349 \\
\hline UTR5 & CNTNAP5 & 0.256273 & 0.445609 & -0.189336 & 0.012985 \\
\hline \multirow[t]{3}{*}{ EXON1 } & PRSS23 & 0.027501 & 0.168661 & -0.14116 & 0.018082 \\
\hline & S100A13 & 0.509213 & 0.660521 & -0.151308 & $1.1 \mathrm{E}-05$ \\
\hline & TIGD5 & 0.112166 & 0.27952 & -0.167354 & 0.001071 \\
\hline \multirow[t]{2}{*}{ GENE BODY } & COQ3 & 0.463529 & 0.637303 & -0.173774 & 0.021585 \\
\hline & MOSC2 & 0.451381 & 0.634923 & -0.183542 & 0.00509 \\
\hline \multirow[t]{3}{*}{ UTR3 } & FLVCR1 & 0.442131 & 0.619624 & -0.177493 & 0.048598 \\
\hline & HLA-B & 0.088913 & 0.308803 & -0.21989 & 0.001479 \\
\hline & $\mathrm{RRH}$ & 0.800984 & 0.629198 & 0.17179 & 0.003695 \\
\hline \multirow[t]{2}{*}{ ISLAND } & chr1:19110720-19110932 & 0.56494 & 0.708782 & -0.143842 & 0.038852 \\
\hline & chr15:22982192-22982410 & 0.93844 & 0.771584 & 0.16686 & 0.035381 \\
\hline \multirow[t]{7}{*}{ NSHELF } & chr12:120933649-120934713 & 0.376657 & 0.609267 & -0.23261 & 0.026168 \\
\hline & chr14:105603310-105603532 & 0.730847 & 0.544063 & 0.18678 & 0.03281 \\
\hline & chr17:35165323-35165983 & 0.636466 & 0.394896 & 0.24157 & 0.009788 \\
\hline & chr18:21083161-21084153 & 0.890993 & 0.690135 & 0.20086 & 0.008755 \\
\hline & chr4:4119800-4120035 & 0.5605 & 0.294444 & 0.26606 & 0.017018 \\
\hline & chr4:47427692-47427905 & 0.54905 & 0.703312 & -0.154262 & 0.04677 \\
\hline & chr4:81951941-81952808 & 0.65228 & 0.810351 & -0.158072 & 0.026402 \\
\hline NSHORE & chr5:178565716-178565918 & 0.721065 & 0.861094 & -0.140029 & 0.008616 \\
\hline \multirow[t]{9}{*}{ SSHELF } & chr1:219786334-219786562 & 0.218291 & 0.432222 & -0.213931 & 0.00867 \\
\hline & chr13:26586287-26586761 & 0.603417 & 0.764495 & -0.161078 & 0.039872 \\
\hline & chr14:71108008-71109332 & 0.876179 & 0.589911 & 0.28627 & 0.00262 \\
\hline & chr16:25268786-25269728 & 0.878063 & 0.59304 & 0.28502 & 0.001536 \\
\hline & chr1:6761295-6762131 & 0.719637 & 0.559159 & 0.16048 & 0.011819 \\
\hline & chr16:85305690-85305892 & 0.365183 & 0.543685 & -0.178502 & 0.045519 \\
\hline & chr22:20459267-20461473 & 0.495691 & 0.667066 & -0.171375 & 0.037325 \\
\hline & chr2:8825106-8826188 & 0.724995 & 0.875096 & -0.1501 & 0.025348 \\
\hline & chr4:120987617-120988156 & 0.859063 & 0.656103 & 0.20296 & 0.000565 \\
\hline \multirow[t]{3}{*}{ SSHORE } & chr10:54631211-54631426 & 0.480326 & 0.686335 & -0.206009 & 0.006475 \\
\hline & chr1:19110720-19110932 & 0.547506 & 0.705954 & -0.158447 & 0.002265 \\
\hline & chr6:169195967-169196186 & 0.577833 & 0.762515 & -0.184682 & 0.010715 \\
\hline
\end{tabular}

DMR differentially methylated region, DR diabetic retinopathy, TSS transcriptional start site, UTR untranslated region, MDFI MyoD family inhibitor, $P G B D 5$ piggyBac transposable element derived 5, ADRA2C adrenoceptor alpha 2C, CNTNAP5 contactin-associated protein-like 5, PRSS23 serine protease 23, S100A13 calcium-binding protein A13, TIGD5 tigger transposable element derived 5, COQ3 coenzyme Q3 methyltransferase, MOSC2 mitochondrial amidoxime-reducing component 2, FLVCR1 feline leukemia virus sub-group $C$ cellular receptor $1, H L A-B$ major histocompatibility complex, class I $B, R R H$ retinal pigment epithelium-derived rhodopsin homolog

\section{Replication study}

The basic and clinical characteristics of the 87 DR and 89 non-DR subjects are summarized in Table 4. Except for DM duration and HbA1C levels, no significant differences were observed in blood glucose levels, BMI, intraocular pressure, equivalent spherical rate, and axial length between the two groups. The 89 participants in the non-DR group did not present signs of DR on annual eye examination after being diagnosed with T2DM. Their disease durations were $>20$ years. 


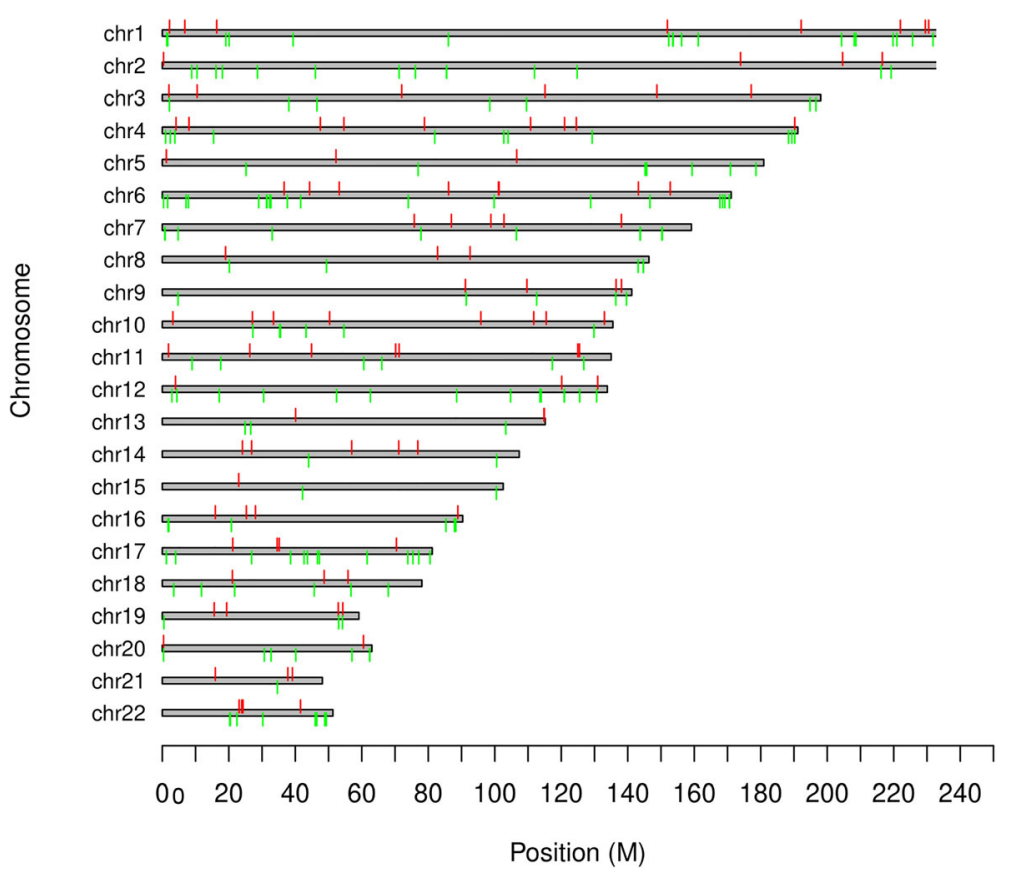

Fig. 1 The distribution of differential methylation sites (DMSs). Red markers represent the hypermethylation sites of the DR group, and green markers represent the hypomethylation site

The association between DR and DMS in the S100A13 gene was verified through pyrosequencing (primer sequences are provided in Supplement 4). Significant differences $(P<0.05)$ between the DR and non-DR groups were observed at sites cg02873163+12 $(41.91 \pm 12.60 \%$; $47.73 \pm 12.36 \%), \operatorname{cg} 02873163+2(54.99 \pm 14.77 \% ; 61.45 \pm$ 13.68\%), cg02873163 (51.03 \pm 14.63\%; $57.21 \pm 13.26 \%$ ), $\operatorname{cg} 11343894+5(45.86 \pm 15.88 \% ; 51.47 \pm 14.34)$, and $\operatorname{cg} 11343894(48.83 \pm 15.17 \% ; 54.51 \pm 14.45)$ in the S100A13 gene. After adjusting for age, sex, BMI, HbA1C, and mean arterial pressure, the average methylation level of all 5 sites of $\operatorname{cg} 02873163$ and $\operatorname{cg} 11343894$ was positively associated with DR through binomial logistic regression analysis (Table 5).

\section{Discussion}

To identify possible epigenetic biomarkers of DR among individuals with T2DM, we enrolled T2DM patients segregated into a discovery cohort and a replication cohort and identified 34 DMRs associated with DR in Chinese individuals with T2DM. These 34 DMRs included 23 (67.65\%) hypomethylation sites, concurrent with a previous report on T1DM patients [5]. DR results from a cascade of enzymatic reactions. With the regulation of DNA methyltransferases and ten-eleven-translocation proteins, DNA hypermethylation/demethylation alters the binding of transcription factors and genes in most cases [12-14]. Interactions between genetic and epigenetic factors may play a role in DR pathogenesis.

Table 3 Genes harboring differential methylation sites (DMSs) with the top 5 differences in $\beta$ values between diabetic retinopathy (DR) and non-DR subjects in the discovery study

\begin{tabular}{|c|c|c|c|c|c|c|c|}
\hline \multirow[t]{2}{*}{ DMS } & \multirow[t]{2}{*}{ Gene } & \multirow[t]{2}{*}{ C } & \multirow[t]{2}{*}{ Location } & \multicolumn{3}{|l|}{$\beta$ value } & \multirow[t]{2}{*}{$P$ value } \\
\hline & & & & DR group & Non-DR group & Difference & \\
\hline cg11596404 & S100A13 & 1 & TSS200; 5' UTR; TSS1500; & 0.530201 & 0.679582 & -0.149381 & $6.39 \mathrm{E}-07$ \\
\hline cg02873163 & S100A13 & 1 & TSS200; 5' UTR; 5' UTR; TSS1500 & 0.550267 & 0.706868 & -0.156601 & $6.42 \mathrm{E}-06$ \\
\hline cg17776284 & S100A13 & 1 & 5' UTR; TSS200; 1stExon; TSS1500 & 0.475831 & 0.621 & -0.145169 & $1.51 \mathrm{E}-05$ \\
\hline cg11915664 & S100A13 & 1 & TSS200; 5' UTR; TSS1500 & 0.410099 & 0.552284 & -0.142185 & $1.55 \mathrm{E}-05$ \\
\hline cg11343894 & S100A13 & 1 & 5' UTR; 1stExon; TSS200;TSS150; & 0.504602 & 0.658721 & -0.154119 & $1.78 \mathrm{E}-05$ \\
\hline
\end{tabular}

DMS differentially methylated site, DR diabetic retinopathy, $C$ chromosome, S100A13 calcium-binding protein A13, TSS transcriptional start site, UTR untranslated region 


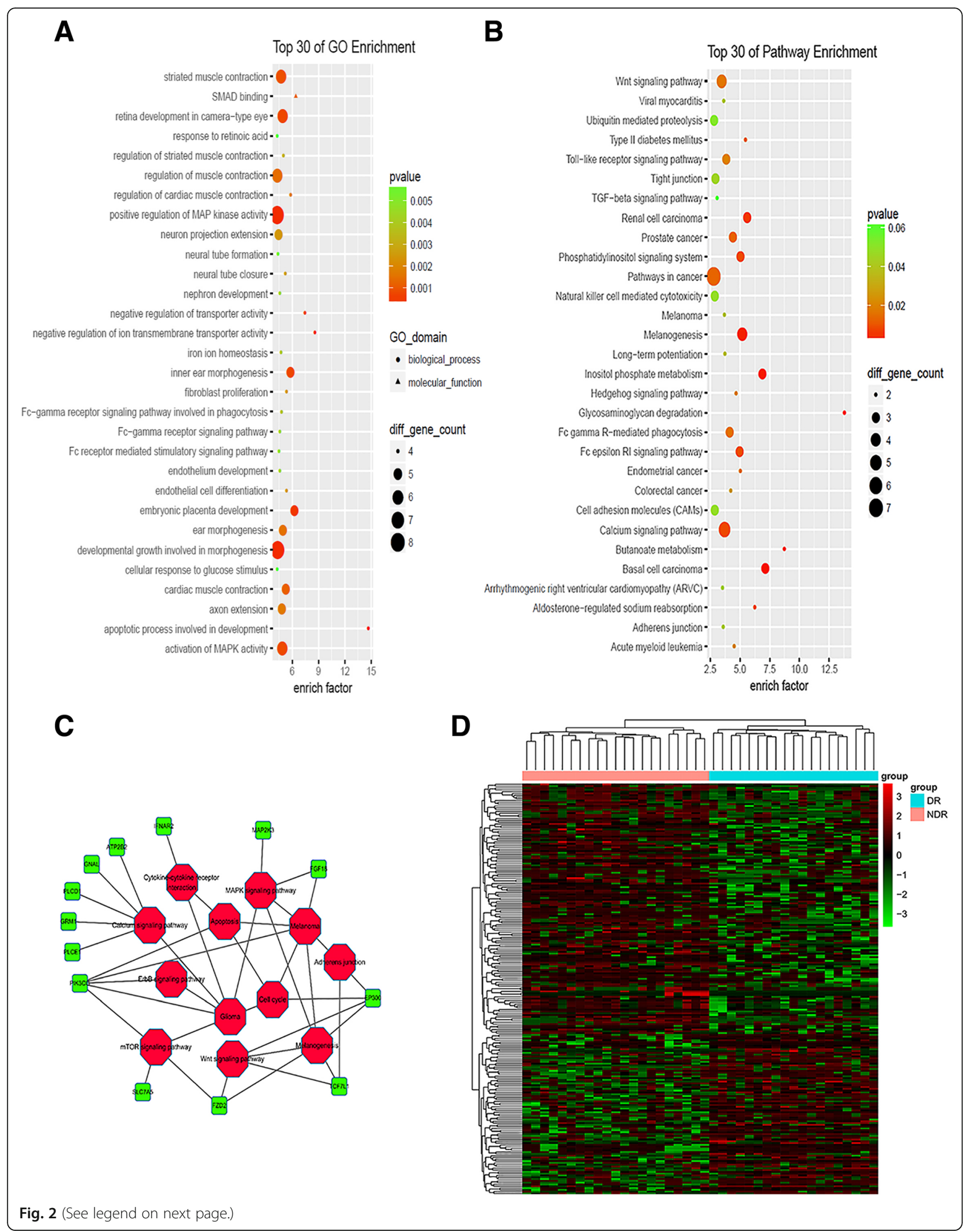


(See figure on previous page.)

Fig. 2 The GO enrichment map of differential methylation sites (DMSs), and the top 30 items are displayed in the graph. The larger the enrichment value is, the more significant the enrichment is. Fig. $2 \mathbf{b}$ shows the KEGG pathway analysis of DMSs in genes, and the top 30 items are displayed in the graph. Figure 2 c shows the potentially most important pathway network, and the differentially methylated genes are involved in pathways of cytokine receptor signaling, MAPK signaling pathway, apoptosis, calcium signaling pathway, adhesion factor, ErbB signaling pathway, cell cycle, Wnt signaling pathway, mTOR pathway, and others. Figure $2 \mathbf{d}$ shows the heat map of DMS in the diabetic retinopathy (DR) group comparing to the non-DR group. The sites are classified to one of the following eight regions: TSS1500 (1500 bp upstream to $200 \mathrm{bp}$ upstream of TSS), TSS200 (200 bp upstream of TSS), 5' UTR, the first exon (1st Exon), 3' UTR, other exon (excluding the first exon), and intronic and intergenic regions. Red indicates DMSs above the average level, and green indicates DMSs below the average of all of the samples

Chen et al. reported that different blood glucose levels affect the extent of DNA methylation in specific genes in whole blood or monocytes, which subsequently affects DR pathogenesis [9]. Herein, blood glucose and HbA1c levels were lower among DR subjects than among nonDR subjects, indicating that the etiology of DR is complex and does not simply depend on glucose levels. We show that the S100A13 gene is potentially involved in DR pathogenesis and that the cg02873163 and cg11343894 sites in the S100A13 gene may serve as potential biomarkers of DR.

The S100A13 gene is involved in calcium ion homeostasis, energy metabolism, inflammation, apoptosis, regulation of proliferation, differentiation, and interactions with transcription factors and nucleic acids within cells and activates surface receptors, including the receptor for advanced glycation end-products and Toll-like receptor 4, G-protein-coupled receptors, scavenger receptors,

Table 4 Basic and clinical characteristics of the 87 diabetic retinopathy patients (type 2 diabetes mellitus duration of $<3$ years) and the 89 patients with no sign of diabetic retinopathy with a type 2 diabetes mellitus duration of $>20$ years enrolled in the replication study

\begin{tabular}{llll}
\hline Characteristics* $^{*}$ & DR group & Non-DR group & $P$ value \\
\hline Number & 87 & 89 & $/$ \\
Sex (male) & 38 & 34 & 0.54 \\
Age (year) & $66.55 \pm 8.27$ & $74.60 \pm 8.80$ & 0.00 \\
Age at DM onset (year) & $64.45 \pm 8.12$ & $48.40 \pm 9.17$ & 0.00 \\
DM duration (year) & $2.10 \pm 0.95$ & $26.19 \pm 4.17$ & 0.00 \\
Blood glucose (mmol/L) & $7.48 \pm 2.44$ & $7.93 \pm 2.51$ & 0.22 \\
HbA1c (\%) & $7.02 \pm 1.37$ & $7.83 \pm 1.36$ & 0.00 \\
BMI (kg/m $\left.{ }^{2}\right)$ & $25.31 \pm 3.98$ & $25.95 \pm 7.03$ & 0.46 \\
MAP(mmHg) & $101.65 \pm 14.23$ & $96.61 \pm 14.06$ & 0.35 \\
SER (D) & $0.04 \pm 2.14$ & $-0.17 \pm 2.22$ & 0.67 \\
IOP (mmHg) & $13.72 \pm 3.51$ & $14.08 \pm 3.83$ & 0.53 \\
AL (mm) & $22.52 \pm 4.44$ & $22.63 \pm 4.65$ & 0.30 \\
\hline DR dabetic &
\end{tabular}

$D R$ diabetic retinopathy, T2DM type 2 diabetes mellitus * $B M l$ body mass index; MAP $(\mathrm{mmHg})$ mean arterial pressure, equal to (systolic pressure $+2 *$ diastolic pressure)/3; SER (D) spherical equivalent rate, equal to spherical power $+1 / 2$ cylindrical power; IOP intraocular pressure; $A L$ axial length or heparan sulfate proteoglycans and N-glycans $[15,16]$. Furthermore, S100A13 regulates calcium levels and fibroblast growth factor-1 and interleukin-1 alpha secretion through a noncanonical pathway $[10,11,17]$. Mandinov et al. reported that S100A13 mediates IL-6induced damage to the macrovasculature [18]. We speculate that significant demethylation of S100A13 in individuals with DR downregulates S100A13 and upregulates p38 MAPK and nuclear factor-kappa B through calcium signaling and the RAGE pathway, thus increasing the damage caused by hyperglycemia [17, 19-23]. Patients harboring hypomethylated S100A13 may require closer follow-up evaluation and more stringent blood glucose screening.

It is generally difficult to confirm a gene-negative contrast group in genetic studies on chronic diseases. For example, DR may occur throughout the lifetime of an individual with T2DM, and the exact diagnosis of nonDR can only be made towards the end of an individual's lifespan. Herein, we selected non-DR individuals with a maximal DM duration to serve as non-DR participants. Furthermore, we selected individuals who developed DR with a DM duration of $<3$ years as the gene-positive group, since we believe that epigenetic changes initiate retinal neural and vascular impairment within a short period. This study cohort displayed large differences in age, onset age, and duration of DM between the two groups, subsequently leading us to speculate whether differences in DNA methylation are associated with age or duration of T2DM. Therefore, we conducted two consecutive studies, including different non-DR contrast groups with different DM durations, and found that

Table 5 Binomial logistic regression analysis of the associations between diabetic retinopathy and methylation levels (\%) at the differential methylation sites

\begin{tabular}{lll}
\hline & Beta $(95 \% \mathrm{Cl})$ & $P$ \\
\hline $\operatorname{cg} 02873163+12(\%)$ & $1.039(1.007,1.072)$ & 0.017 \\
cg02873163+2(\%) & $1.033(1.005,1.061)$ & 0.020 \\
cg02873163 (\%) & $1.034(1.006,1.063)$ & 0.019 \\
cg11343894+5(\%) & $1.025(1.000,1.051)$ & 0.048 \\
cg11343894(\%) & $1.026(1.000,1.053)$ & 0.046 \\
\hline
\end{tabular}


DMSs in the S100A13 gene were present in both studies; moreover, the associations between DMSs and DR were confirmed with regression analysis.

This study has several limitations. First, subjects developing DR within a relatively short duration or those without DR in the long term may harbor gene mutations, which potentially affect approximately $14 \%$ of the methylation levels at corresponding or adjacent sites [24]. Second, each cell type has a different DNA methylation programming system [18]. DMSs in whole blood cells can only reflect the potential for the occurrence of diabetic vascular disease instead of duplicate DMSs in retinal cells. Finally, 22 DMRs are present in CpG islands located distal to any known gene among the 34 DMRs, and their functions warrant further elucidation in future studies.

\section{Conclusions}

Our study is the first to detect DMRs and DMSs associated with DR in T2DM patients, indicating that DMSs in the S100A13 gene serve as potential biomarkers of DR. Future studies with more genetically diverse cohorts having a shorter DM duration of $<10$ years and prospective cohort studies are required to confirm the effect of epigenetic changes in the S100A13 gene on DR incidence.

\section{Methods}

This retrospective study is based on the Shanghai Xinjing community prevention and treatment system of chronic diseases, which was established in 1999. The system has curated annually updated health data on all local residents diagnosed with T2DM. In 2003, our group participated in this system, and we have been conducting and supervising studies on the prevention and treatment of ocular diseases [25-29]. From June to August 2016, we conducted an epidemiological study on eye complications of DM upon receiving ethical approval by the Ethics Committee of Shanghai General Hospital, Shanghai Jiaotong University School of Medicine (2013KY023). This study describes the epigenetic analysis conducted within that study. This study adheres to the tenets of the Helsinki Declaration, and the study subjects provided written informed consent to participate in the study.

The inclusion criteria were as follows: (1) Chinese Han background, (2) a diagnosis of T2DM in accordance with the World Health Organization criteria [30], and (3) the ability to comply with all the required examinations. The exclusion criteria were as follows: (1) occurrence of eyelid diseases, strabismus, corneal diseases, lens diseases, and other eye diseases potentially affecting the outcomes of retinal examinations; (2) occurrence of other eye diseases, including glaucoma or macular degeneration, which may cause fundus retinal microvasculopathy; (3) history of eye surgery or trauma; (4) occurrence of severe systemic diseases, including those involving the respiratory system, circulatory system, and excretory system; and (5) history of cancer.

The research staff were fully trained and experienced with epidemiologic studies [25-27]. We gathered data including demographic characteristics and conducted a routine eye examination. DR was diagnosed on the basis of the well-accepted international diagnostic criteria. For each participant, $2 \mathrm{~mL}$ samples of fasting blood were obtained, the cell composition was determined for further data analysis, and DNA was extracted using a QIAN amp blood kit (Qiagen, Hilden, Germany) in accordance with the manufacturer's instructions.

\section{Discovery study}

\section{Enrolled participants}

The discovery cohort included 19 subjects who developed DR with a DM duration of $<3$ years and 21 subjects without $\mathrm{DR}>30$ years after being diagnosed with DM.

\section{DNA methylation quantification}

The genomic DNA (500 ng) was subjected to bisulfite conversion using an EZ DNA methylation kit (Zymo Research, Orange, CA, USA) in accordance with the manufacturer's instructions. An Infinium Human Methylation 850 Beadchip methylation chip (Illumina, San Diego, CA, USA), which covers $99 \%$ of all RefSeq genes and contains 867,531 sites, was used. Methylation analysis involved data quality control, preprocessing, methylation difference site analysis, and methylation difference region analysis.

The quality control step for the analysis of the $850 \mathrm{k}$ chip included checking for sex (X,Y-chromosome data were not included in the DMS/DMR analysis) [31], filtering the SNP sites (minor allele frequency $>5 \%$ and probes with SNPs of minor allele frequency $>5 \%$ within 10 base pairs of the CpG sites were excluded) [32], (list of CpG sites is available at http://genetics. emory.edu/research/conneely/annotation-r-code.html), normalization (Subset-quantile Within Array Normalization was used to normalize the DNA methylation probe intensity) [33], following the criteria for retaining probes (detection $P<$ 0.05 in more than half of the samples) and individuals (detection $P$ value of more than $95 \%$ of the probes of each sample $<0.05)$.

\section{Replication study Enrolled participants}

Pyrosequencing was performed to verify the correlation between the candidate DMSs and DR. The replication cohort included 87 subjects who developed DR with a 
DM duration of $<3$ years and 89 subjects without DR over a DM duration $>20$ years.

\section{Pyrosequencing}

DNA (500 ng) was subjected to bisulfite conversion with the EZ DNA methylation kit (Zymo Research) in accordance with the manufacturer's instructions. Pyrosequencing was performed with the PyroMark Q96 ID system (Qiagen, Valencia, CA, USA) in accordance with the manufacturer's recommendations. The PyroMark PCR Master Mix kit (Qiagen), streptavidin-coated beads (GE Healthcare, Uppsala, Sweden), PyroMark Gold Q96 reagents (Qiagen), PyroMark Q96 Vacuum Workstation, and PyroMark Q96 software (version 2.5.8, Qiagen) were used to determine and analyze DMSs.

\section{Data processing and statistical analysis}

The clinical data were statistically analyzed using the SPSS 22.0 software (IBM Corporation, Armonk, NY, USA). The data are expressed as the mean \pm standard deviation values, and calculated values are presented as frequencies and percentages. Data normality was assessed using the Kolmogorov-Smirnov test. Normally distributed data were analyzed using Student's $t$-test to compare differences in various values between the DR and non-DR groups. Skewed data were analyzed using the Mann-Whitney test. Statistical tests performed herein were the chi-square test and independent samples $t$ test.

Minfi software package (version 1.25.1, http://www. bioconductor.org/packages/release/bioc/html/minfi.

html) and IMA software package (version 2.0, http:// www.rforge.net/IMA), both written with $\mathrm{R}$ software, were used to determine the differences in the degree of DNA methylation between the DR participants and the non-DR participants [34]. Minfi package was used to calculate the beta value (interval between 0 and 1), an indicator of the degree of methylation using the following equation: $\quad$ beta $_{\mathrm{i}}=\frac{\max \left(y_{(\mathrm{i}, \text { methy }}, 0\right)}{\max \left(y_{(\mathrm{i}, \mathrm{meth})}, 0\right)+\max \left(y_{(\mathrm{i}, \text {,unmethy }}, 0\right)+100}$. The distribution of the beta values was skewed, and all outlier beta values were excluded (omitted values $>4 \mathrm{SD}$ ).

The sitetest algorithm and the regionswrapper algorithm, both from the IMA package, were used to define DMS and DMR, respectively. Du et al. suggested the threshold of beta value as between 0.05 and 0.15 [35], and according to the Illumina's instructions, 0.14 is the minimum value recommended to guarantee the proper sensitivity. Therefore, if differences between beta values at a certain region or site between the DR and non-DR groups were greater than 0.14 and the $P$ value determined via a pooled $t$ test was less than 0.05 , the region was considered a DMR, and the site was considered a DMS without regression analysis.
The functions of genes containing DMRs or DMSs were assessed via KEGG enrichment analysis, GO analysis, and pathway network analysis. We mapped the methylated sites to GO terms in the Gene Ontology database and determined and corrected (Bonferroni) the number of genes for each GO term. KEGG pathway analysis (http://www.kegg.jp/) was performed to confirm the enriched pathways. The threshold for statistical significance $(<0.05)$ was defined on the basis of the corrected $P$ value. The gene coexpression network was constructed in accordance with the normalized signal intensity to identify gene interactions. A strong correlation (Pearson correlation $>0.9$ ) indicated the interaction between two genes. Cytoscape (National Institute of General Medical Sciences, Boston, MA, USA) was used to construct coexpression networks. If a gene harbored a DMS with higher differences in beta values between DR and nonDR participants and the corresponding protein was involved in previously reported pathways associated with DR pathogenesis, the DMS was considered a candidate DMS in the following replication study. The $\mathrm{R}$ 3.5.1 pheatmap package was used to calculate and draw the heat map.

In the replication part, the association between methylation level (\%) on pyrosequencing and DR was adjusted for demographic characteristics, including age, sex, Hb1Ac (\%), body mass index, and mean arterial pressure, which were assessed through binomial logistic regression analysis. A $P$ value $<0.05$ was considered statistically significant.

\section{Supplementary information}

Supplementary information accompanies this paper at https://doi.org/10. 1186/s13148-020-00871-z.

Additional file 1:. Supplement 1.

Additional file 2:. Supplement 2.

Additional file 3:. Supplement 3.

Additional file 4:. Supplement 4.

Abbreviations

DR: Diabetic retinopathy; T2DM: Type 2 diabetes mellitus; DMG: Differential methylation region; DMS: Differential methylation site; GO: Gene Ontology; S100A13: Calcium-binding protein A13; T1DM: Type 1 DM; HbA1c: Hemoglobin A1C; BMl: Body mass index; MAP: Mean arterial pressure; SER: Spherical equivalent rate; IOP: Intraocular pressure; AL: Axial length; TSS: Transcription start site; UTR: Untranslated region; MDFI: MyoD family inhibitor; PGBD5: PiggyBac transposable element derived 5;

ADRA2C: Adrenoceptor alpha 2C; CNTNAP5: Contactin-associated protein-like 5; PRSS23: Serine protease 23; S100A13: Calcium-binding protein A13; TIGD5: Tigger transposable element derived 5; COQ3: Coenzyme Q3 methyltransferase; MOSC2: Mitochondrial amidoxime reducing component 2; FLVCR1: Feline leukemia virus sub-group C cellular receptor 1; HLA-B: Major histocompatibility complex, class I B; RRH: Retinal pigment epitheliumderived rhodopsin homolog; C: Chromosome 


\section{Acknowledgements}

We appreciate the assistance and cooperation of staffs from Shanghai Eye Diseases Prevention \& Treatment Center and Shanghai Xinjing Community health center.

\section{Authors' contributions}

$\mathrm{TL}$ and JC undertook DNA extraction and methylation measurement in samples. TL undertook the analyses and wrote the manuscript with supervision from $H Z$. TL, YX, YS, JZ, SL, LL, and XX were major contributors to data collection and interpretation. $Y X, X X, L L, J Z$, and $H Z$ took charge of the epidemiological study and the sample collection. $\mathrm{HZ}$ and $\mathrm{TL}$ were responsible to explain the data on which the paper is based. The final paper has been read and approved by all authors. $\mathrm{HZ}$ is the guarantors.

\section{Funding}

Chinese National Nature Science Foundation (Project number 81670898), The Project of Shanghai Shen Kang Hospital Development Centre (Grant No. SHDC2018110), Shanghai Municipal Education Commission—Gaofeng Clinical Medicine Grant Support (Project No. 20172022), Shanghai Engineering Center for Precise Diagnosis and Treatment of Eye Diseases (Project No. 19DZ2250100)

\section{Availability of data and materials}

The datasets used and/or analyzed during the current study are available from the corresponding author on reasonable request.

\section{Ethics approval and consent to participate}

The study was approved by the Ethics Committee of the First People's Hospital affiliated to Shanghai Jiaotong University (2013KY023). This study conformed to the guidelines proposed in the Helsinki Convention. After a full explanation of the entire study process, each subject signed an informed consent form at the start of the study.

\section{Consent for publication}

Not applicable

\section{Competing interests}

The authors declare that they have no competing interests.

\section{Author details}

'Shanghai Eye Diseases Prevention \& Treatment Center/Shanghai Eye Hospital, No. 380, Kangding Road, Shanghai 200040, China. ${ }^{2}$ Department of Ophthalmology, Shanghai General Hospital, Shanghai Jiaotong University School of Medicine, Shanghai, China. ${ }^{3}$ Bio-X Institutes, Key Laboratory for the Genetics of Developmental and Neuropsychiatric Disorders (Ministry of Education), the Collaborative Innovation Center for Brain Science, Shanghai Jiaotong University, Shanghai, China. ${ }^{4}$ Shanghai Key Laboratory of Psychotic Disorders, Shanghai Mental Health Center, Shanghai Jiaotong University School of Medicine, Shanghai, China.

\section{Received: 7 January 2020 Accepted: 21 May 2020}

\section{Published online: 03 June 2020}

\section{References}

1. Youngson NA, Morris MJ. What obesity research tells us about epigenetic mechanisms. Philos Trans R Soc Lond Ser B Biol Sci. 2013;1(5):368 (1609)

2. Goldberg $A D$, Allis $C D$, Bernstein E. Epigenetics: a landscape takes shape. Cell. 2007;128(4):635-8.

3. Mishra M, Kowluru RA. The role of DNA methylation in the metabolic memory phenomenon associated with the continued progression of diabetic retinopathy. Invest Ophthalmol Vis Sci. 2016;57(13):5748-57.

4. Kowluru RA, Shan Y, Mishra M. Dynamic DNA methylation of matrix metalloproteinase-9 in the development of diabetic retinopathy. Lab Investig. 2016:96(10):1040-9.

5. Agardh E, Lundstig A, Perfilyev A, Volkov P, Freiburghaus T, Lindholm E, Ronn T, Agardh CD, Ling C. Genome-wide analysis of DNA methylation in subjects with type 1 diabetes identifies epigenetic modifications associated with proliferative diabetic retinopathy. BMC Med. 2015;6(13):182

6. Dos Santos Nunes MK, Silva AS, de Queiroga Evangelista IW, Filho JM, Gomes C, do Nascimento RAF, Luna RCP, de Carvalho Costa MJ, de Oliveira NFP, Persuhn DC. Hypermethylation in the promoter of the MTHFR gene is associated with diabetic complications and biochemical indicators. Diabetol Metab Syndr. 2017;18(9):84

7. Mishra M, Zhong Q, Kowluru RA. Epigenetic modifications of Keap1 regulate its interaction with the protective factor Nrf2 in the development of diabetic retinopathy. Invest Ophthalmol Vis Sci. 2014;55(11):7256-65.

8. Klein BE. Overview of epidemiologic studies of diabetic retinopathy. Ophthalmic Epidemiol. 2007;14(4):179-83.

9. Chen Z, Miao F, Paterson AD, Lachin JM, Zhang L, Schones DE, Wu X, Wang J, Tompkins JD, Genuth S, Braffett BH, Riggs AD, Natarajan R. Epigenomic profiling reveals an association between persistence of DNA methylation and metabolic memory in the DCCT/EDIC type 1 diabetes cohort. Proc Natl Acad Sci U S A. 2016;113(21):E3002-11.

10. Su Y, Xu C, Sun Z, Liang Y, Li G, Tong T, Chen J. S100A13 promotes senescence-associated secretory phenotype and cellular senescence via modulation of non-classical secretion of IL-1alpha. Aging. 2019;11(2):549-72.

11. Landriscina M, Schinzari G, Di Leonardo G, Quirino M, Cassano A, D'Argento E, Lauriola L, Scerrati M, Prudovsky I, Barone C. S100A13, a new marker of angiogenesis in human astrocytic gliomas. J Neuro-Oncol. 2006;80(3):251-9.

12. Ling C, Groop L. Epigenetics: a molecular link between environmental factors and type 2 diabetes. Diabetes. 58(12):2718-25.

13. Jaenisch $\mathrm{R}$, Bird $\mathrm{A}$. Epigenetic regulation of gene expression: how the genome integrates intrinsic and environmental signals. Nat Genet. 2003; 33(Suppl):245-54.

14. Jones PA. Functions of DNA methylation: islands, start sites, gene bodies and beyond. Nat Rev Genet. 2012;13(7):484-92.

15. Hofmann MA, Drury S, Fu C, Qu W, Taguchi A, Lu Y, Avila C, Kambham N, Bierhaus A, Nawroth P, Neurath MF, Slattery T, Beach D, McClary J, Nagashima M, Morser J, Stern D, Schmidt AM. RAGE mediates a novel proinflammatory axis: a central cell surface receptor for S100/calgranulin polypeptides. Cell. 1999;97(7):889-901.

16. Chazin WJ. Relating form and function of EF-hand calcium binding proteins. Acc Chem Res. 2011:44(3):171-9.

17. Al-Mutairy EA, Imtiaz FA, Khalid M, Al Qattan S, Saleh S, Mahmoud LM, AlSaif MM, Al-Haj L, Al-Enazi A, AlJebreen AM, Mohammed SF, Mobeireek AF, Alkattan K, Chisti MA. An atypical pulmonary fibrosis is associated with coinheritance of mutations in the calcium binding protein genes S100A3 and S100A13. Eur Respir J. 2019;54(1).

18. Bacos K, Gillberg L, Volkov P, Olsson AH, Hansen T, Pedersen O, Gjesing AP, Eiberg H, Tuomi T, Almgren P, Groop L, Eliasson L, Vaag A, Dayeh T, Ling C. Blood-based biomarkers of age-associated epigenetic changes in human islets associate with insulin secretion and diabetes. Nat Commun. 2016;7: 11089.

19. Rani SG, Mohan SK, Yu C. Molecular level interactions of S100A13 with amlexanox: inhibitor for formation of the multiprotein complex in the nonclassical pathway of acidic fibroblast growth factor. Biochemistry. 2010; 49(11):2585-92.

20. Rani SG, Sepuru KM, Yu C. Interaction of S100A13 with C2 domain of receptor for advanced glycation end products (RAGE). Biochim Biophys Acta. 2014;1844(9):1718-28.

21. Donato R. RAGE: a single receptor for several ligands and different cellular responses: the case of certain S100 proteins. Curr Mol Med. 2007;7(8):71124.

22. Li C, Zhang F, Wang Y. S100A proteins in the pathogenesis of experimental corneal neovascularization. Mol Vis. 2010;16:2225-35.

23. Cao R, Yan B, Yang H, Zu X, Wen G, Zhong J. Effect of human S100A13 gene silencing on FGF-1 transportation in human endothelial cells. J Formos Med Assoc. 2010;109(9):632-40.

24. Kulkarni H, Kos MZ, Neary J, Dyer TD, Kent JW Jr, Goring HH, Cole SA, Comuzzie AG, Almasy L, Mahaney MC, Curran JE, Blangero J, Carless MA. Novel epigenetic determinants of type 2 diabetes in Mexican-American families. Hum Mol Genet. 2015;24(18):5330-44

25. Xu X, He J, Xu X, Du X, Bai X, Zhang B, He X, Lu L, Zhu J, Zou H. Cataract was the principle cause of visual impairment and blindness in Shanghai residents with type 2 diabetes. Acta Ophthalmol. 2016;94:e246-7.

26. Zou X, Lu L, Xu Y, Zhu J, He J, Zhang B, Zou H. Prevalence and clinical characteristics of dry eye disease in community-based type 2 diabetic patients: the Beixinjing eye study. BMC Ophthalmol. 2018;18:117.

27. He J, Xu X, Zhu J, Zhu B, Zhang B, Lu L, He X, Bai X, Xu X, Zou H. Lens power, axial length-to-corneal radius ratio, and association with diabetic retinopathy in the adult population with type 2 diabetes. Ophthalmology. 2017; 124:326-35. 
28. Wang N, Xu X, Zou H, Zhu J, Wang W, Ho PC. The status of diabetic retinopathy and diabetic macular edema in patients with type 2 diabetes: a survey from Beixinjing District of Shanghai city in China. Ophthalmologica. 2008;222(1):32-6.

29. Huang XB, Zou HD, Wang N, Wang WW, Fu J, Shen BJ, Xie TH, Chen YH, Xu $X$, Zhang $X$. A prevalence survey of blindness and visual impairment in adults aged equal or more than 60 years in Beixinjing blocks of Shanghai, China. Chin J Ophthalmol. 2009:45:786-92.

30. Petersmann A, Nauck M, Muller-Wieland D, Kerner W, Muller UA, Landgraf R, Freckmann G, Heinemann L. Definition, classification and diagnosis of diabetes mellitus. Exp Clin Endocrinol Diabetes. 126(7):406-10.

31. Paul DS, Teschendorff AE, Dang MA, et al. Increased DNA methylation variability in type 1 diabetes across three immune effector cell types. Nat Commun. 2016;7:13555.

32. Barfield RT, Almli LM, Kilaru V, et al. Accounting for population stratification in DNA methylation studies. Genet Epidemiol. 2014;38:231-41.

33. Maksimovic J, Gordon L, Oshlack A. SWAN: subset-quantile within array normalization for illumina infinium Human Methylation450 BeadChips. Genome Biol. 2012;13(6):R44.

34. Yu H, Du L, Yi S, et al. Epigenome-wide association study identifies Behçet's disease associated methylation loci in Han Chinese. Rheumatology (Oxford). 2019:58(9):1574-84.

35. Du P, Zhang X, Huang CC, et al. Comparison of beta-value and M-value methods for quantifying methylation levels by microarray analysis. BMC Bioinformatics. 2010;11:587.

\section{Publisher's Note}

Springer Nature remains neutral with regard to jurisdictional claims in published maps and institutional affiliations.

Ready to submit your research? Choose BMC and benefit from:

- fast, convenient online submission

- thorough peer review by experienced researchers in your field

- rapid publication on acceptance

- support for research data, including large and complex data types

- gold Open Access which fosters wider collaboration and increased citations

- maximum visibility for your research: over $100 \mathrm{M}$ website views per year

At $\mathrm{BMC}$, research is always in progress.

Learn more biomedcentral.com/submissions 